\title{
Multidisciplinary Approach to Nanostructure Determination
}

\author{
Christian Dwyer ${ }^{1}$
}

1. Department of Physics, Arizona State University, Tempe, Arizona, USA

It is becoming increasingly apparent that the next era of transmission electron microscopy will arguably lie within the realm of "data analysis". This follows the most-recent decades of "aberration correction", in which progress in leaps and bounds were made in our ability to form high-resolution images using electron beams. The data analysis era will grow out of a necessity for extracting progressively weaker signals in our quest for understanding the nanoworld in ever greater detail. The ideas involved are intrinsically multidisciplinary, invoking input from the fields of signal processing, electron scattering theory, and condensed matter modeling (in addition of course to carefully-controlled quantitative experiments in electron microscopy). The era of data analysis has the potential to transform transmission electron microscopy, from a science of "picture taking", to one capable of performing quantitative measurements of weak signals, akin to what is done in the fields of advanced astronomy and particle physics.

In this talk, I will discuss some steps towards this direction, in which quantitative experiments, electron scattering calculations, density functional theory calculations and image processing techniques are used to understand the energetics of precipitates in advanced aluminium alloys. In this body of work [1-5], quantitative imaging of the atomic structures in judiciously-chosen crystallographic orientations is performed using annular dark-field (ADF) STEM. This mode of imaging is selected for its robustness in the presence of dynamical scattering, its sensitivity to changes in chemical composition (atomic number) and its amenability to accurate quantification through frozen-phonon multislice calculations. As a natural by product, images of precipitates contain regions of the surrounding matrix where the atomic structure is accurately known. Such regions permit calibration of the ADF intensity, as well as measurement of the sample thickness, which can also be checked against convergent-beam diffraction patterns.

The atomic-resolution ADF images then serve as an excellent starting part for structure refinement of the precipitate phase. The positions of intensity peaks often provide an excellent indication of the atomic column positions, while peak intensity can be used as a measure of atomic-number density. In some case, the peak "shape" can also give indications on the presence of localized strain. In the latter cases, competing effects can make refinement is extremely challenging, though it is often still possible to determine structural parameters (e.g. occupancies) to within reasonable bounds. Structural refinement, including bounds on structural parameters, can then proceed using accurate frozen-phonon multislice calculations in an iterative manner.

Throughout the structural refinement process, density functional theory calculations can play a crucial role. The accuracy and reliability of these calculations in many alloy systems means that they can be used to determine whether a proposed structure is energetically plausible. In some cases, we have found that relaxation of an input structure has lead to significant structural changes, which, in turn, have produced far better agreement between our experimental and multislice-simulated images. In this sense, density functional theory is a tool that very much complements the quantitative experiments, and can even provide feedback into the experimental process. 
Fig. 1a shows an exemplary experimental and simulated ADF-STEM image of a $\mathrm{T}_{1}$ precipitate in an Al$\mathrm{Li}-\mathrm{Cu}$ alloy. In our original work [1], the procedure above was used to refine the atomic structure (overlaid) including the occupancies of fractionally occupied atomic sites at the bounding planes and middle plane of the precipitates. Here, we have performed new density functional calculations, based on a special-quasirandom-structure approach, as a means of gauging the plausibility of these measured occupancies in the bounding planes, and the comparison between theory and experiment gives reasonable agreement. We have also performed density functional calculations for a selection of the various $T_{1}$ structures proposed in previous decades, confirming that our determined structure is the most energetically favorable. For example, as shown in Fig. 1b, our model gives the lowest energy structure, in contrast to the previously accepted structure of Huang and Ardell, which proves to be energetically unstable.

The present examples illustrate the power of a multidisciplinary approach to nanostructure determination based on electron microscopy, electron scattering theory, density functional theory and image processing.

References:

[1] C Dwyer, M Weyland, LY Chang, and BC Muddle, Applied Physics Letters 98 (2011), 201909.

[2] L Bourgeois, C Dwyer, M Weyland, J-F Nie, and BC Muddle, Acta Materialia 59 (2011), 7043.

[3] R Bjørge, C Dwyer, M Weyland, PNH Nakashima, CD Marioara, SJ Andersen, J Etheridge, and R Holmestad, Acta Materialia 60 (2012), 3239.

[4] L Bourgeois, NV Medhekar, AE Smith, M Weyland, JF Nie, and C Dwyer, Physical Review Letters 111 (2013) 046102.

[5] JM Rosalie, C Dwyer, and L Bourgeois, Acta Materialia 69 (2014) 224.

(a)

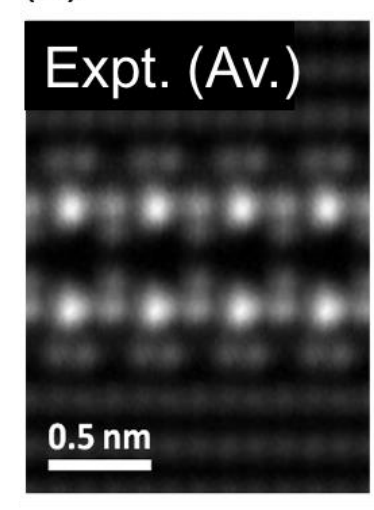

(b)

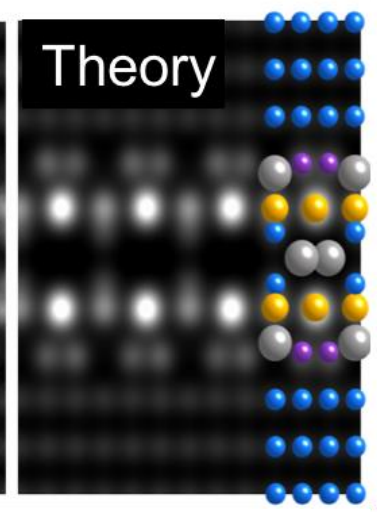

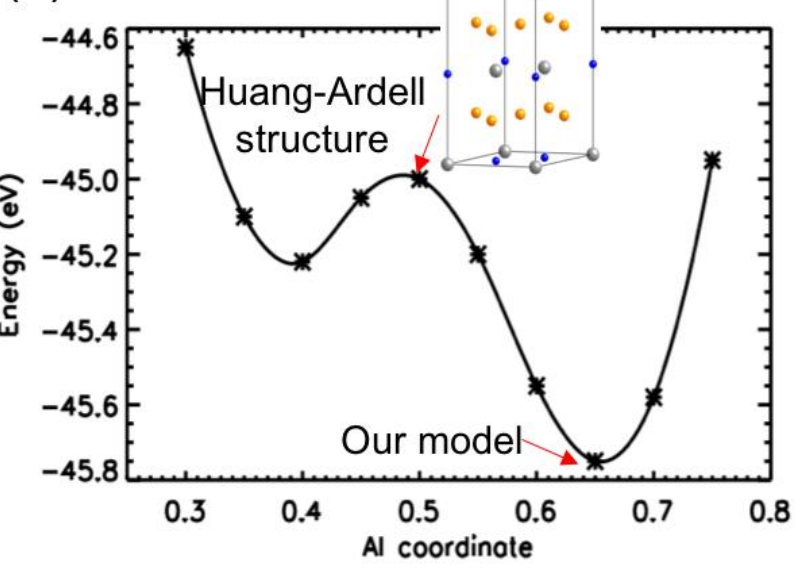

Figure 1. (a) Experimental and simulated HAADF-STEM image of a $\mathrm{T}_{1}$ precipitate in an Al-Li-Cu alloy. The atomic model is overlaid. (b) The energetics of the previously-accepted Huang-Ardell model of $\mathrm{T}_{1}$ precipitates compared to our model. 